\title{
Transparent Metasurface for Generating Microwave Vortex Beams with Cross-Polarization Conversion
}

\author{
Hongyu Shi ${ }^{1}{ }^{\circledR}$, Luyi Wang ${ }^{1}{ }^{\circledR}$, Mengran Zhao ${ }^{1}{ }^{\oplus}$, Juan Chen ${ }^{2, *}$, Anxue Zhang ${ }^{1}$ and Zhuo Xu ${ }^{3}$ \\ 1 School of Electronic and Information Engineering, Xi'an Jiaotong University, Xi'an 710049, China; \\ honyo.shi1987@gmail.com (H.S.); bigcrash@stu.xjtu.edu.cn (L.W.); zmr1993@stu.xjtu.edu.cn (M.Z.); \\ anxuezhang@xjtu.edu.cn (A.Z.) \\ 2 Xi'an Jiaotong University Shenzhen Research School, Shenzhen 518057, China \\ 3 Electronic Materials Research Laboratory, Key Laboratory of the Ministry of Education, \\ Xi'an Jiaotong University, Xi'an 710049, China; xuzhuo@xjtu.edu.cn \\ * Correspondence: chen.juan.0201@xjtu.edu.cn
}

Received: 25 October 2018; Accepted: 26 November 2018; Published: 3 December 2018

\begin{abstract}
In this paper, metasurfaces with both cross-polarization conversion and vortex beam-generating are proposed. The proposed finite metasurface designs are able to change the polarization of incident electromagnetic (EM) waves to its cross-polarization. In addition, they also can modulate the incidences into beams carrying orbital angular momentum (OAM) with different orders $(l=+1, l=+2, l=-1$ and $l=-2)$ by applying corresponding transmission phase distribution schemes on the metasurface aperture. The generated vortex beams are at $5.14 \mathrm{GHz}$. The transmission loss is lower than $0.5 \mathrm{~dB}$ while the co-polarization level is $-10 \mathrm{~dB}$ compared to the cross-polarization level. The measurement results confirmed the simulation results and verified the properties of the proposed designs.
\end{abstract}

Keywords: vortex beam; polarization conversion; orbital angular momentum

\section{Introduction}

The orbital angular momentum of electromagnetic waves has been explored in recent years for its potential applications in wireless communications [1-3] and imaging [4,5]. EM wave carrying orbital angular momentum has a helical wavefront and an amplitude singularity in the propagating direction. The helical wavefront can be expressed by the term $\exp (i l \Phi)$, where $\Phi$ is the azimuthal angle and $l$ is the topological charge. The topological charge corresponds to the OAM mode and, theoretically, the OAM mode is vast.

The OAM in EM waves is typically generated using techniques like spiral phase plates [6,7], spiral reflectors [8], antennas [9-11], dieletric resonators [12], computer-induced holograms [13], transformation electromegnetics [14] and metasurfaces $[15,16]$. The common idea in these techniques is to introduce the desired phase distribution on the radiation aperture. The spiral phase plate method gives the incident wave different phase retardation according to the term $\exp (i l \Phi)$ by modulating the length of the wave path in corresponding areas. The antenna array approach usually use a circular antenna array to excite array elements with the same amplitude but different phases.

Compared to these methods, metasurfaces for generation of beams carrying OAM have advantages including low profile and simple EM wave control, i.e., the magnitude/phase/polarization of the EM waves can be manipulated simply by changing the shape, geometry, size, orientation and arrangement of the structures [17]. Reflective metasurfaces were used to generate single and double mode vortex beams in mircrowave [18-21] and terahertz regimes [22]. An active transparent metasurface was proposed for generating EM beams carrying OAM in the microwave frequency 
range [23]. However, these designs only focus on the OAM controlling of microwaves leaving the polarization state of the transmitted wave the same as that of the incidence. Simultaneously control the polarization and OAM of EM waves can enhance the performances of OAM beams in applications like radar imaging. Recently, metasurfaces using Geometric-Phase were applied for simultaneous OAM and spin angular momentum control [24-26]. These techniques impart a new degree of freedom to EM wave control and pave a way for future applications.

In this paper, multi-layered metasurfaces generating OAM beams with efficient linear polarization conversion were proposed. The patches on the outer sides of the designed metasurface receive and re-radiate the incident wave, respectively. The cross-polarized transmission is higher than $-1 \mathrm{~dB}$ around $5.15 \mathrm{GHz}$ with an extremely low co-polarized transmission below $-35 \mathrm{~dB}$. The transmission phase can be fully controlled by the length of the stripline in the middle layer. By arranging the metasurface unit cells according to desired phase distributions, the proposed metasurfaces can generate EM beams carrying different modes of OAM. This design method was demonstrated by both simulation and measurement.

This paper is organized as follows: Section 2 presents the design of the unit cells and the metasurfaces. In this section, detailed geometries of the unit cell are introduced, the characteristics of the unit cell are shown and the metasurface designs are presented. Section 3 presents the simulated and measured results of the metasurfaces, which verify the designs and show OAM generation with polarization conversion. In Section 4 , the conclusions are drawn and the originality of this work is presented.

\section{Design of The Metasurface}

A flowchart illustrating the main goal and the adopted methodology of this study is presented in Figure 1. The unit cell pattern of the proposed multi-layered laminated metasurface is depicted in Figure 2, where the gray parts represent the substrate Rogers $4350 \mathrm{~B}$ with $\epsilon_{r}=3.48$ and $\tan \delta=0.0037$. The yellow parts represent the metal structures with a thickness of $0.035 \mathrm{~mm}$. The top and bottom layers of the metasurface are circular patches which can couple or decouple the incident wave with a cross-polarization conversion. The middle layer of the metasurface is a stripline structure and is separated from the top and bottom layers by the first and second ground layers, respectively. Two vias connect the two ends of the stripline to the top and bottom layers, respectively. The geometric dimensions are $p=17.92 \mathrm{~mm}, R=16.64 \mathrm{~mm}, r=0.8 \mathrm{~mm}, w=1.2 \mathrm{~mm}, f_{x}=f_{y}=3 \mathrm{~mm}, h_{1}=1.524 \mathrm{~mm}$ and $h_{2}=0.254 \mathrm{~mm}$. The length of the stripline $S$ varies from $1.66 \mathrm{~mm}$ to $8.49 \mathrm{~mm}$ to achieve a $360^{\circ}$ transmission phase control.

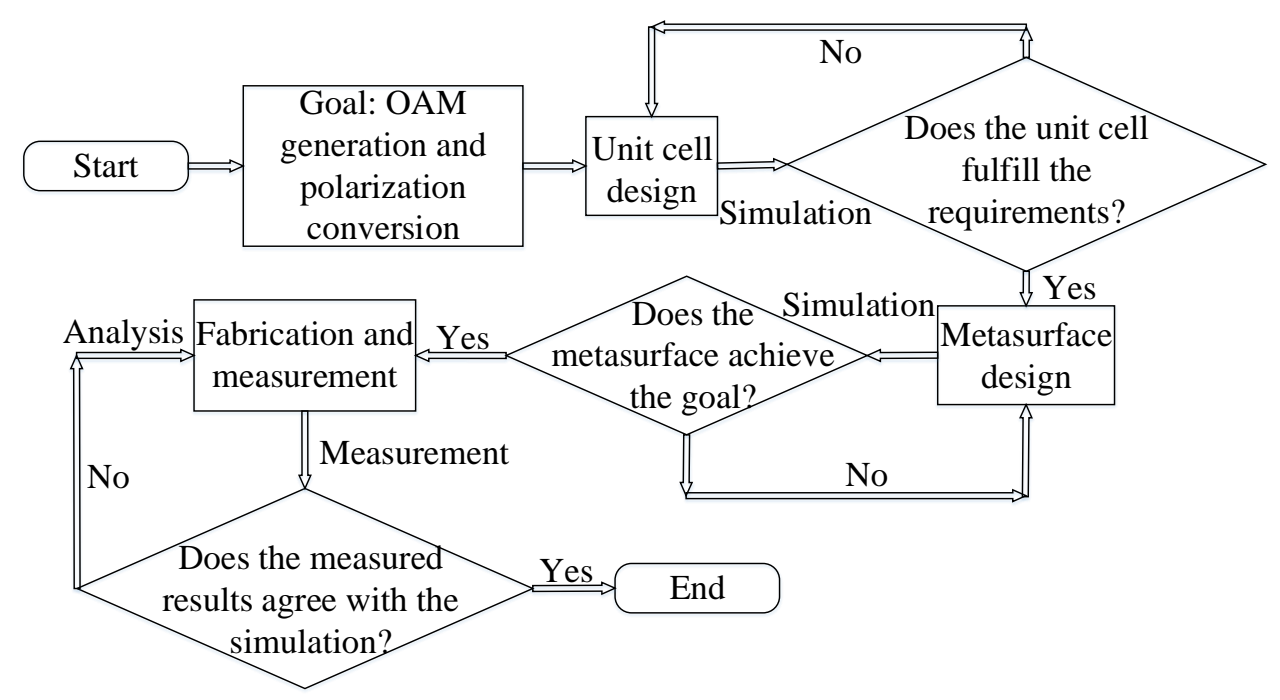

Figure 1. Flowchart illustrating the main goal and the adopted methodology of this paper. 

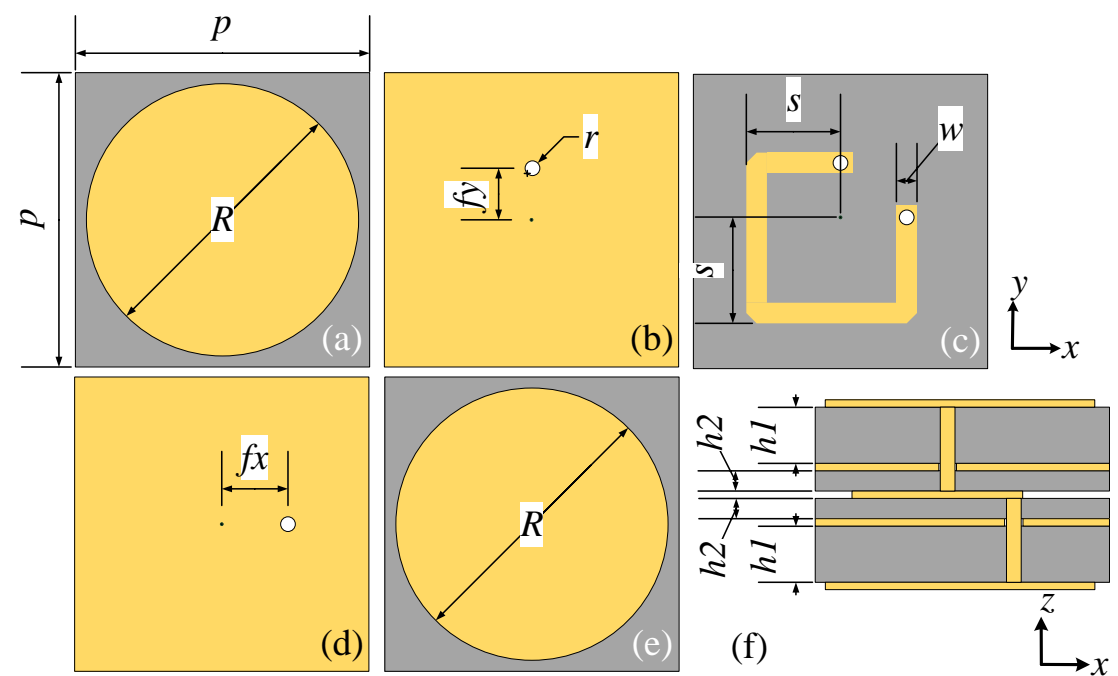

Figure 2. Geometry of the unit cell: (a) Top layer. (b) First ground layer. (c) Middle layer. (d) Second ground layer. (e) Bottom layer. (f) Side view.

The unit cell design takes its inspiration from the patch antenna. The top/bottom layer couples the $y$-polarized/x-polarized incident wave into the guided mode in the stripline structure and then, the bottom/top layer decouples the guided wave into the $x$-polarized/y-polarized free space propagation. It is by selecting the positions of the vias in orthogonal direction (e.g., in $x$ and $y$ directions), that the polarization of the transmitted wave is converted.

The simulated distributions of the electric field component perpendicular to the unit cell (i.e., $\mathrm{E}_{z}$ ) on top and bottom layers are shown in Figure 3a,b, respectively. The incidences excite a y-polarized dipolar mode on the top layer, where the guided mode travels through the stripline structure to the bottom layer and excite a x-polarized dipolar mode, therefore converting the polarization of the transmitted waves. In addition, the guided mode experiences different phase delay when the length of the stripline varies. Also, the energy loss in the stripline structure is small and consistent regardless of its lengths. Therefore, the transmission phase can be controlled by the length of the stripline, which allows different phase distributions for different OAM beam-generating, while the transmission loss is small and stable. Notably, due to only the top and bottom layers resonate, this design has potentials to obtain low insert loss.

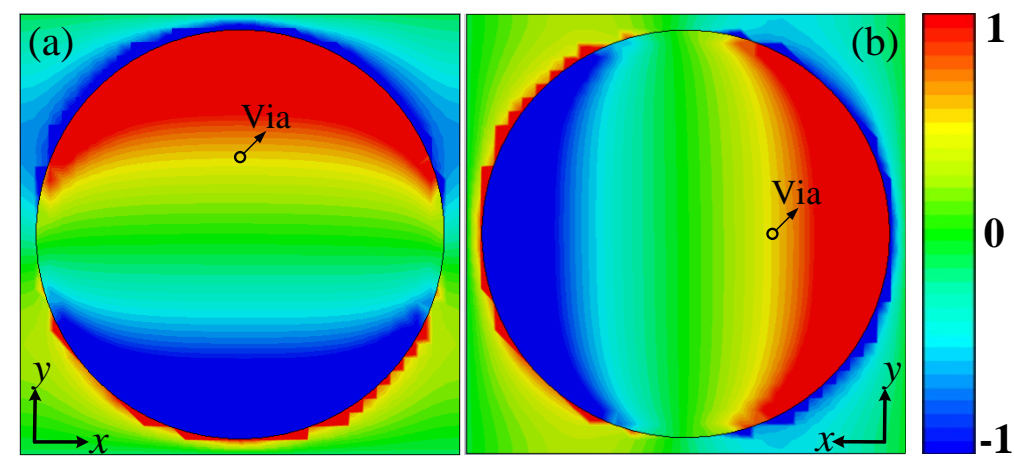

Figure 3. The simulated distributions of the electric field component perpendicular to the unit cell (i.e., $\mathrm{E}_{z}$ ): (a) Top layer. (b) Bottom layer.

The unit cell models were simulated by the commercial software CST Microwave Studio (Version 2016, Computer Simulation Technology GmbH, Darmstadt, Germany) using periodic boundary in $x$ and $y$ directions. The simulated transmission amplitudes and phases are shown in Figure $4 \mathrm{a}, \mathrm{b}$, respectively. The transmission phase data at $4.8-5.5 \mathrm{GHz}$ are given because, at other 
frequency ranges, the curves are confused and not of main concern in this paper. For a $y$-polarized incidence propagating along $-z$ direction, the transmitted wave is $x$-polarized and the cross-polarized transmission amplitudes with different $S$ are higher than $-0.5 \mathrm{~dB}$ at $5.14 \mathrm{GHz}$, and from $4.9 \mathrm{GHz}$ to $5.4 \mathrm{GHz}$, the transmittances are higher than $-3 \mathrm{~dB}$ which indicates a $50 \%$ power efficiency, as shown in Figure 4a. The co-polarized transmittances are below $-35 \mathrm{~dB}$ from $4.9 \mathrm{GHz}$ to $5.4 \mathrm{GHz}$, indicating an extremely high polarization conversion efficiency, compared with $[27,28]$. At $5.14 \mathrm{GHz}$, the co-polarized transmittance is below $-38.2 \mathrm{~dB}$. Eight stripline lengths were selected with a transmission phase step of $45^{\circ}$ to cover a $360^{\circ}$ phase difference, as shown in Figure $4 \mathrm{~b}$. The selected stripline lengths are $1.656 \mathrm{~mm}, 2.62 \mathrm{~mm}, 3.62 \mathrm{~mm}, 4.58 \mathrm{~mm}, 5.57 \mathrm{~mm}, 6.54 \mathrm{~mm}, 7.53 \mathrm{~mm}$ and $8.49 \mathrm{~mm}$.

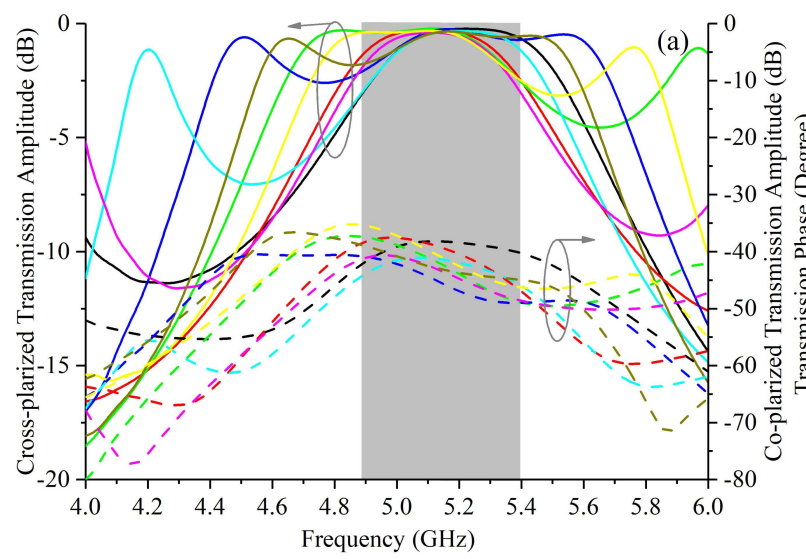

Stripline Length $S=1.66 \mathrm{~mm}-2.62 \mathrm{~mm}$

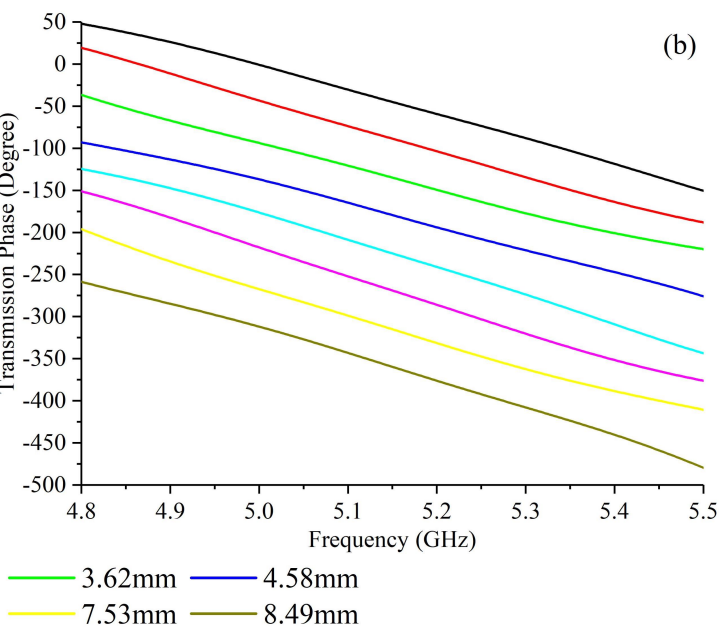

(b)

Figure 4. The simulated transmittance of the unit cell with different stripline lengths $S$ (as in Figure 2c): (a) Amplitude. (b) Phase.

The helical wavefront of vortex beams can be expressed by the term $\exp (i l \Phi)$, where $\Phi$ is the azimuthal angle and $l$ is the topological charge. Therefore, EM beams carrying OAM with an order of $l$ experiences an azimuthal phase change of $|l| \times 360^{\circ}$. The sign of the OAM order $l$ defines the helicity of the vortex beam phase distribution. To generate vortex beam-carrying OAM of orders \pm 1 and \pm 2 , two transmission phase distributions at $5.14 \mathrm{GHz}$ with phase steps of $45^{\circ}$ and $90^{\circ}$, respectively were designed and shown in Figure 5a,b, which depict the desired transmission phase with regard to different positions on metasurfaces. For wave propagating along $-z$ and $z$ directions, these two designs have opposite helicities and generate EM beams carrying OAM with orders of $1 / 2$ and $-1 /-2$, respectively.

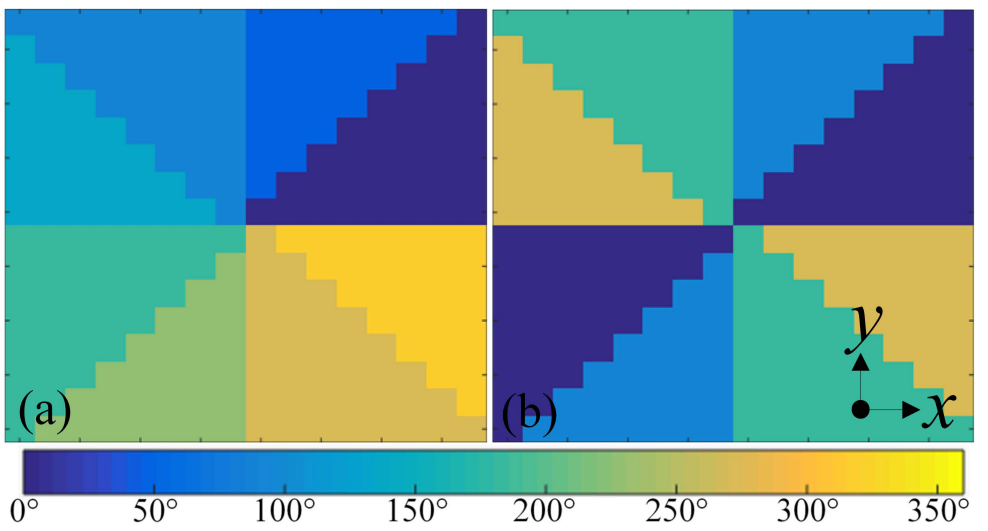

Figure 5. The front view of the transmission phase distribution schemes at $5.14 \mathrm{GHz}$ for generating beams carrying OAM of different orders: (a) $l=+1$. (b) $l=+2$. 
The two finite full structure models containing $16 \times 16$ unit cells are shown in Figure 6 . The discretization of the metsurface is done according to the transmission phase distributions in Figure 5. The target frequency of the metasurfaces is at $5.14 \mathrm{GHz}$. It is worth pointing out that the potential applications for radar imaging can be in X/C/S band and the metasurface design can be easily tuned to other frequencies as well. The models were built up and simulated by CST Microwave studio with a Gaussian beam as excitation with a minimum beam radius of $100 \mathrm{~mm}$ on the metasurface. The average simulation time in a server with 256 GB memory and Intel Xeon E5 CPU is about 6 h. About 30 GB memory is used. Gaussian beam, compared with plane wave, reduces the slight amount of diffractions of the EM waves at the margins, while the phase profile of the transmitted beams are the same. Also, the margins of the metasurfaces have metal sheet in ground layers to further avoid diffractions. The used unit cells for realizing the desired transmission phase distribution designs in Figure $5 \mathrm{a}, \mathrm{b}$ with phase steps of $45^{\circ}$ and $90^{\circ}$ respectively are selected from Figure $4 \mathrm{~b}$. Eight kinds of unit cell with stripline lengths of $1.656 \mathrm{~mm}, 2.62 \mathrm{~mm}, 3.62 \mathrm{~mm}, 4.58 \mathrm{~mm}, 5.57 \mathrm{~mm}, 6.54 \mathrm{~mm}, 7.53 \mathrm{~mm}$ and $8.49 \mathrm{~mm}$ are selected for Figure 5 a while four kinds of unit cell with stripline lengths of $1.656 \mathrm{~mm}$, $3.62 \mathrm{~mm}, 5.57 \mathrm{~mm}$ and $7.53 \mathrm{~mm}$ are selected for Figure $5 \mathrm{~b}$.

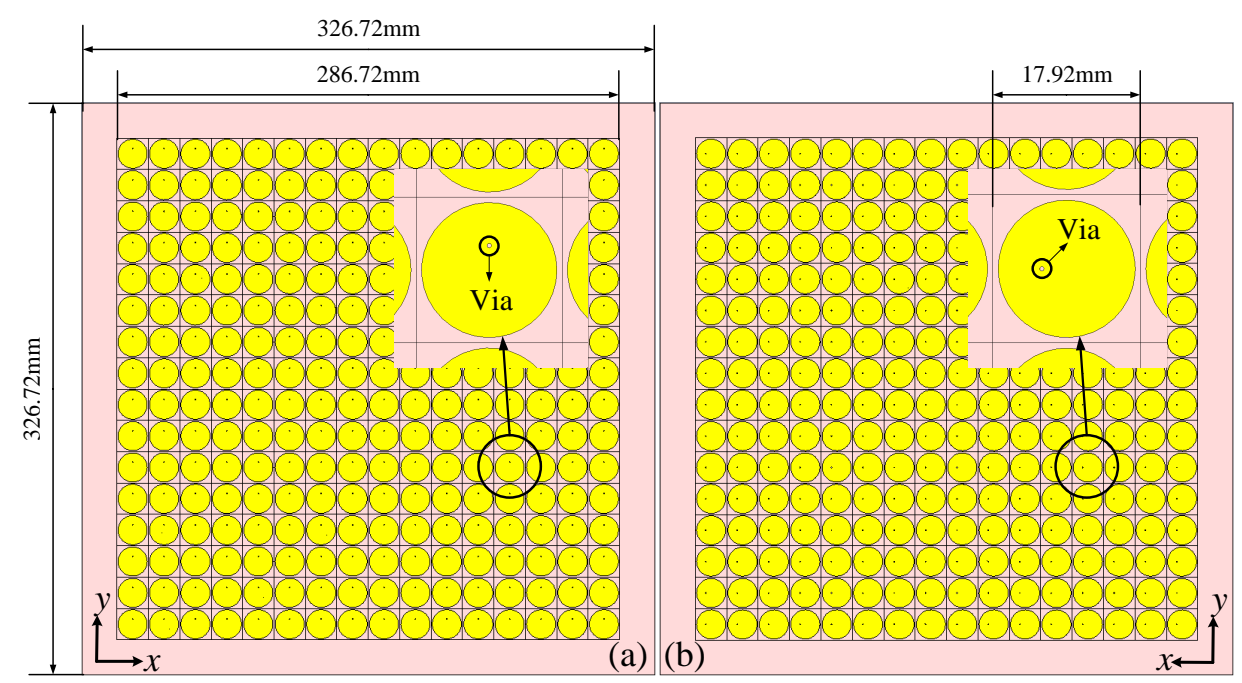

Figure 6. The simulation model of the proposed metasurface: (a) Front view. (b) Back view.

\section{Results}

The simulated transmitted electric field distributions at a transverse plane $250 \mathrm{~mm}$ away from the metasurface are depicted in Figure 7. Figure 7a-d,e-h show the transmitted electric field distributions with incidences propagating along $-z$ direction with a $y$-polarization and along $z$ direction with a $x$-polarization, respectively. Figure $7 \mathrm{a}, \mathrm{c}, \mathrm{e}, \mathrm{g}$ show the normalized transmitted electric field amplitude distributions at 5.14 GHz with design schemes in Figure 5a,b, respectively. Amplitude nulls can be observed due to the phase singularity at the center of OAM carrying beams, and along with the donut-shaped field distribution verified the characteristic of the vortex beams.

The transmitted phase distributions are shown in Figure $7 \mathrm{~b}, \mathrm{~d}, \mathrm{f}, \mathrm{h}$. The phase accumulations along a full circular path around the beam null in Figure $7 b, d$ are $2 \pi$ and $-2 \pi$, which indicates OAM orders of +1 and -1 , respectively. Figure $7 \mathrm{f}$,h depict $4 \pi$ and $-4 \pi$ phase accumulations along a full circular path and therefore indicate OAM orders of +2 and -2 . Thus, by using the proposed structure, the designed metasurfaces can simultaneously convert the polarization of the incident wave and generate vortex beams carrying OAM of four different orders, which has great potentials for radar imaging applications. 

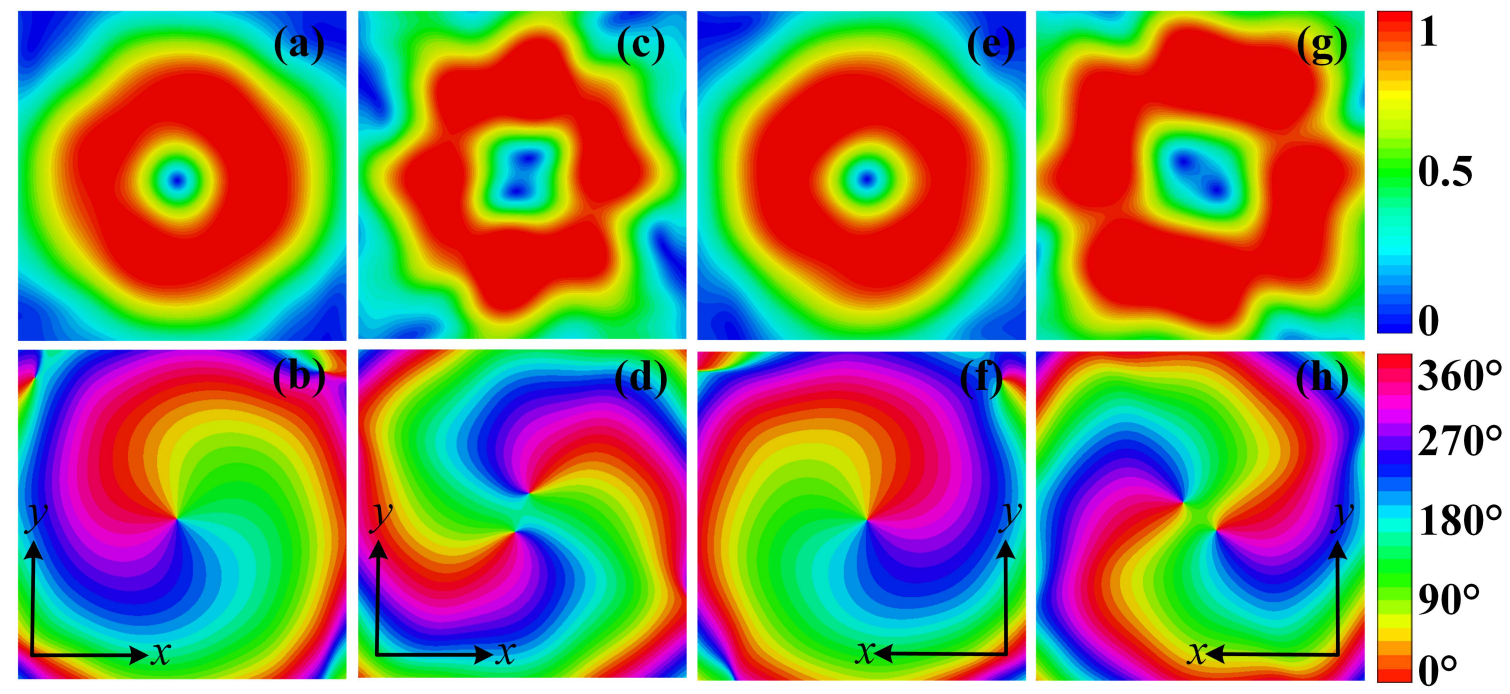

Figure 7. Simulated cross-polarized electric field distributions of the transmitted OAM carrying beams at a transverse plane $250 \mathrm{~mm}$ away from the metasurface: (a) Amplitude and (b) phase distributions for OAM order of $l=+1$. (c) Amplitude and (d) phase distributions for OAM order of $l=+2$. (e) Amplitude and (f) phase distributions for OAM order of $l=-1$. (g) Amplitude and (h) phase distributions for OAM order of $l=-2$.

The proposed metasurface was fabricated using PCB processing as shown in Figure 8. The overall size of the fabricated sample is $326.72 \mathrm{~mm} \times 326.72 \mathrm{~mm}$ with a thickness of $4.35 \mathrm{~mm}$. Vias connecting the middle layer to the top and bottom layers are fabricated by back drilling leaving two holes on the top and bottom layers of each unit cell. The back drill holes and prepregs have been considered in the simulations and have little influences on the metasurfaces performance.

The fabricated metasurfaces were measured using a vector network analyzer Agilent E8363b (Keysight Technologies, California, United States) and a two-dimensional near field scanning measurement system. The metasurface was placed between a lens horn antenna (used as the excitation) and a WR-229 open-ended rectangular waveguide probe (used for receiving the OAM carrying beams). The measurement was conducted in the anechoic chamber. The response calibration was used to eliminate the effect of external noise during the measurement. The polarization conversion was confirmed by receiving and analyzing the co-polarization and cross-polarization components of the EM waves, which was realized by rotating the open-ended rectangular waveguide probe. A schema of the measurement devices and settings is depicted in Figure 9.
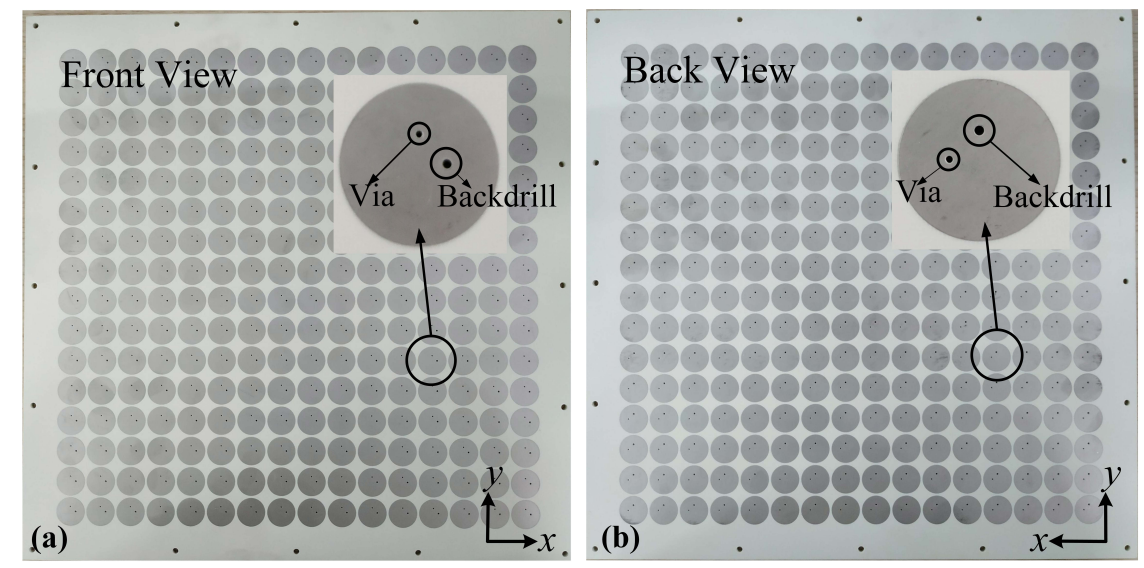

Figure 8. Photos of the fabricated metasurface: (a) Front view. (b) Back view. 


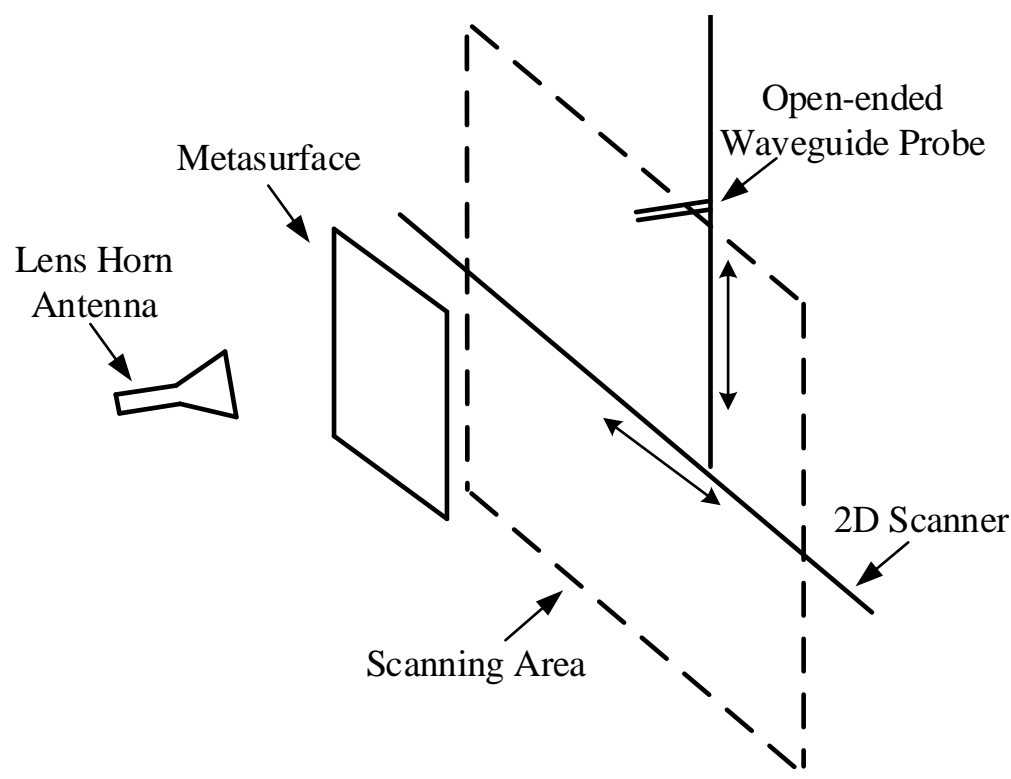

Figure 9. Schema depicting the measurement devices and settings.

The measured amplitude and phase distributions of the cross-polarized transmitted electric field at a transverse plane $250 \mathrm{~mm}$ away from the metasurface are shown in Figure 10. Figure 10a-d show the amplitudes and phases of the $x$-polarized transmitted wave with a $y$-polarized excitation propagating along $-z$ direction. Figure $10 \mathrm{a}, \mathrm{c}$ depict an amplitude null at the field center. Figure $10 \mathrm{~b}, \mathrm{~d}$ show phase accumulations of $2 \pi$ and $4 \pi$ along a full circular path, indicating OAM orders of +1 and +2 , respectively. Figure 10e-h show the amplitudes and phases of the $y$-polarized transmitted wave with an $x$-polarized excitation propagating along $z$ direction. The amplitude distributions shown in Figure 10e,g show amplitude nulls level at 0.01 compared to the maximum value. Due to the deviations in fabrication and measurement, the perfect offset of amplitude at the center may be compromised. Still, $-20 \mathrm{~dB}$ nulls level is satisfying [29]. The phase distributions in Figure 10f,h show $-2 \pi$ and $-4 \pi$ phase accumulations along a full circular path, indicating OAM order of -1 and -2 , respectively.
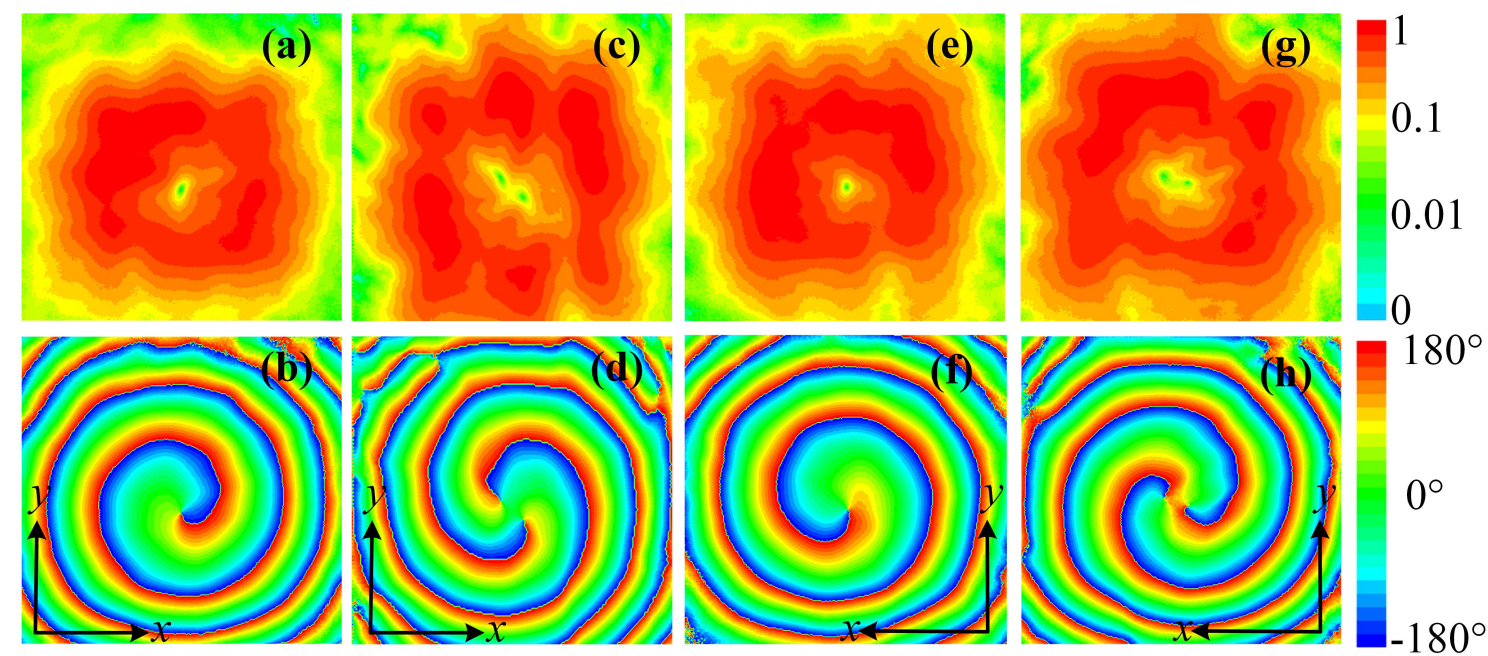

Figure 10. Measured cross-polarized electric field distributions of the transmitted OAM carrying beams at a transverse plane $250 \mathrm{~mm}$ away from the metasurface: (a) Amplitude and (b) phase distributions for OAM order of $l=+1$. (c) Amplitude and (d) phase distributions for OAM order of $l=+2$. (e) Amplitude and (f) phase distributions for OAM order of $l=-1$. (g) Amplitude and (h) phase distributions for OAM order of $l=-2$. 
The simulated and measured amplitudes of the co-polarized transmitted wave are shown in Figure 11a,b, respectively. For each condition, the co-polarized transmissions are randomly distributed with a low amplitude. In the simulation results, the co-polarized electric fields amplitude level is lower than 0.06 , while the measured results show co-polarization level lower than 0.15 . The discrepancy between simulation and measurement comes from fabrication deviations and the slight amount of diffracted EM waves. However, compared with the cross-polarization level, the co-polarization level is low and does not affect the generated cross-polarized vortex beams. The co-polarization level can be enhanced if absorbers are placed around the metasurface.
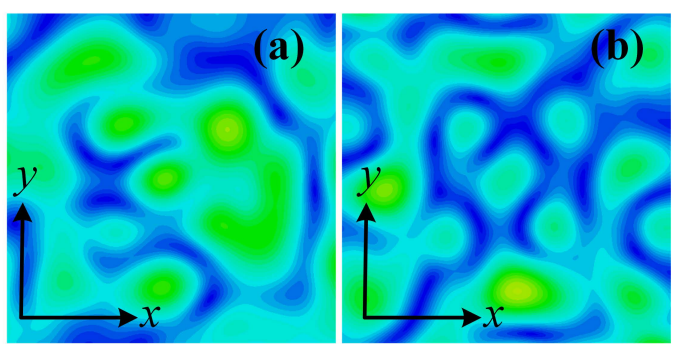

(e)
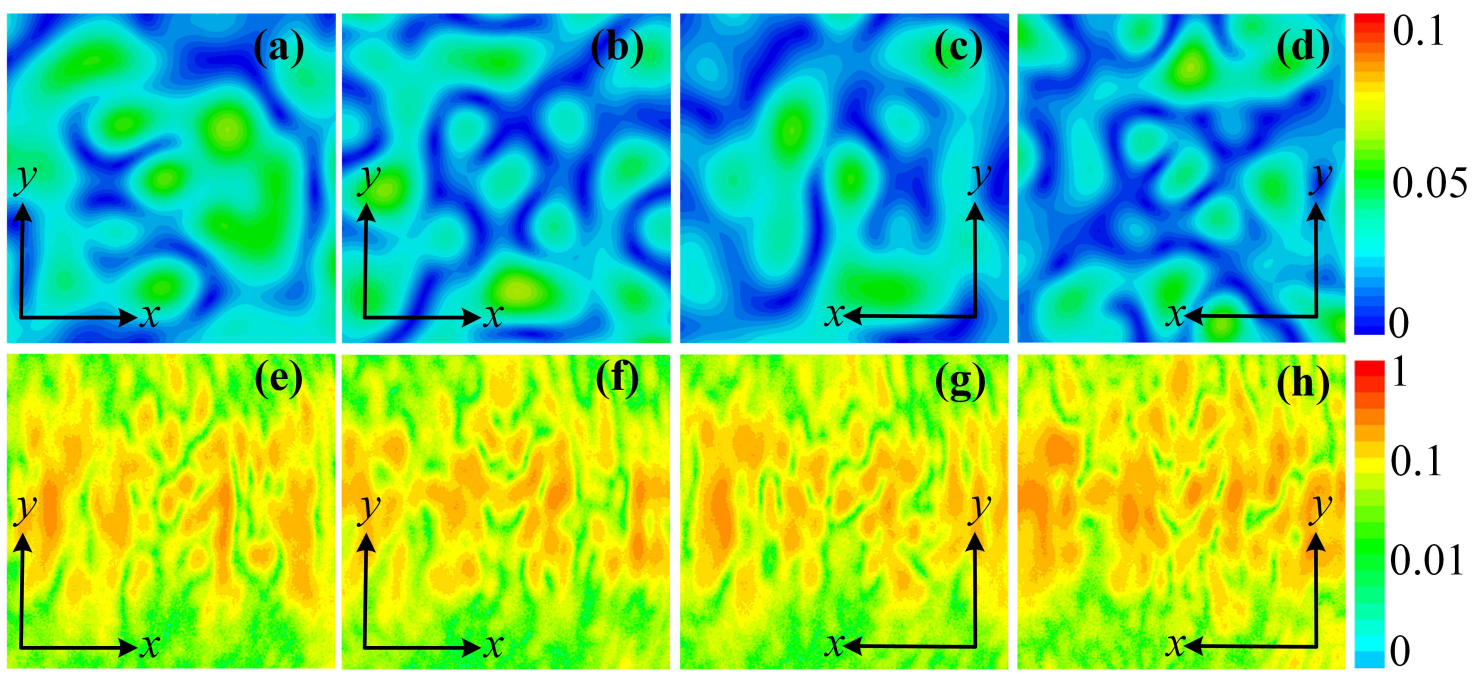

(g)

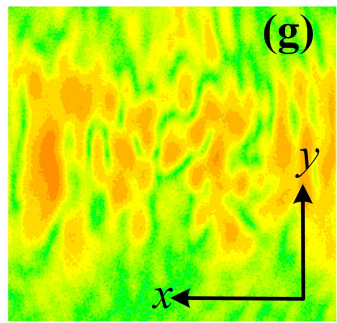

(h)

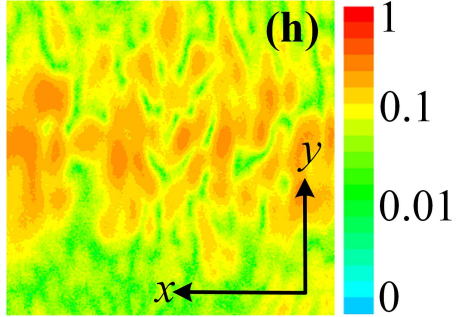

Figure 11. Simulated co-polarized electric fields amplitude distributions for different OAM orders: (a) $l=+1$. (b) $l=+2$. (c) $l=-1$. (d) $l=-2$. Measured co-polarized electric fields amplitude distributions for different OAM orders: (e) $l=+1$. (f) $l=+2$. (g) $l=-1$. (h) $l=-2$.

\section{Conclusions}

In conclusion, two polarization conversion metasurfaces generating four different orders of OAM carrying beams $(l=+1, l=+2, l=-1$ and $l=-2)$ were designed and fabricated. The simulation and measurement results are in good agreement with each other. The multi-layered unit cells we proposed realize full phase control, low transmission loss, high polarization conversion efficiency and can be easily tuned to any frequencies of interest. By manipulating the transmission phase distributions on the metasurface aperture, the transmitted beams can carry OAM of four different orders, which has potentials for super resolution imaging. In addition, the polarizations of the transmitted waves were efficiently converted, which may further enhance the performances in applications, for example, imaging polarization dependent objects.

Author Contributions: Conceptualization, H.S.; methodology, H.S. and L.W.; validation, H.S.; formal analysis, H.S. and L.W.; investigation, L.W. and M.Z.; resources, A.Z.; data curation, L.W.; writing-review and editing, H.S.; visualization, H.S. and L.W.; supervision, H.S. and J.C.; project administration, J.C., A.Z. and Z.X.; funding acquisition, H.S.

Funding: This research was funded by National Natural Science Foundation of China grant number 61871315, in part by Technology Program of Shenzhen grant number JCYJ20170816100722642, in part by the Natural Science Foundation of Guangdong Province, China grant number 2018A030313429 and in part by the China Postdoctoral Science Foundation under Grant 2015M580849. The APC was funded by 61871315.

Conflicts of Interest: The authors declare no conflict of interest. 


\section{References}

1. Yan, Y.; Xie, G.D.; Lavery, M.P.J.; Huang, H.; Ahmed, N.; Bao, C.J.; Ren, Y.X.; Cao, Y.W.; Li, L.; Zhao, Z.; et al. High-capacity millimetre-wave communications with orbital angular momentum multiplexing. Nat. Commun. 2014, 5, 4876. [CrossRef] [PubMed]

2. Hui, X.N.; Zheng, S.L.; Chen, Y.L.; Hu, Y.P.; Jin, X.F.; Chi, H.; Zhang, X.M. Multiplexed Millimeter Wave Communication with Dual Orbital Angular Momentum (OAM) Mode Antennas. Sci. Rep. 2015, 5, 10148. [CrossRef] [PubMed]

3. Mahmouli, F.E.; Walker, S.D. 4-Gbps Uncompressed Video Transmission over a 60-GHz Orbital Angular Momentum Wireless Channel. IEEE Wirel. Commun. Lett. 2013, 2, 223-226. [CrossRef]

4. Liu, K.; Cheng, Y.Q.; Yang, Z.C.; Wang, H.Q.; Qin, Y.L.; Li, X. Orbital-Angular-Momentum-Based Electromagnetic Vortex Imaging. IEEE Antennas Wirel. Propag. Lett. 2015, 14, 711-714. [CrossRef]

5. Liu, K.; Cheng, Y.Q.; Gao, Y.; Li, X.; Qin, Y.L.; Wang, H.Q. Super-resolution Radar Imaging Based on Experimental OAM Beams. Appl. Phys. Lett. 2017, 116, 164102. [CrossRef]

6. Cheng, L.; Hong, W.; Hao, Z.C. Generation of Electromagnetic Waves with Arbitrary Orbital Angular Momentum Modes. Sci. Rep. 2014, 5, 4814. [CrossRef]

7. Chen, Y.; Zheng, S.; Li, Y.; Hui, X.; Jin, X.; Chi, H.; Zhang, X. A Flat-Lensed Spiral Phase Plate Based on Phase-shifting Surface for Generation of Millimeter-wave OAM Beam. IEEE Antennas Wirel. Propag. Lett. 2015, 15, 1156-1158. [CrossRef]

8. Hui, X.; Zheng, S.; Hu, Y.; Xu, C.; Jin, X.; Chi, H.; Zhang, X. Ultralow Reflectivity Spiral Phase Plate for Generation of Millimeter-wave OAM Beam. IEEE Antennas Wirel. Propag. Lett. 2015, 14, 4876. [CrossRef]

9. Bai, Q.; Tennant, A.; Allen, B. Experimental Circular Phased Array for Generating OAM Radio Beams. Electron. Lett. 2014, 50, 1414-1415. [CrossRef]

10. Mohammadi, S.M.; Daldorff, L.K.S.; Bergman, J.E.S.; Karlsson, R.L.; Thide, B.; Forozesh, K.; Carozzi, T.D.; Isham, B. Orbital Angular Momentum in Radio-A System Study. IEEE Trans. Antennas Propag. 2010, 58, 565-572. [CrossRef]

11. Barbuto, M.; Trotta, F.; Bilotti, F.; Toscano, A. Circular Polarized Patch Antenna Generating Orbital Angular Momentum. Prog. Electromagn. Res. 2014, 148, 23-30. [CrossRef]

12. Ren, J.; Leung, K.W. Generation of microwave orbital angular momentum states using hemispherical dielectric resonator antenna. Appl. Phys. Lett. 2018, 112, 131103. [CrossRef]

13. Chavez-Cerda, S.; Padgett, M.J.; Allison, I.; New, G.H.C.; Gutierrez-Vega, J.C.; O’Neil, A.T.; MacVicar, I.; Courtial, J. Holographic generation and orbital angular momentum of high-order Mathieu beams. J. Opt. B-Quantum Semicl. Opt. 2002, 4, S52-S57. [CrossRef]

14. Feng, R.; Yi, J.J.; Burokur, S.N.; Kang, L.; Zhang, H.L.; Werner, D.H. Orbital angular momentum generation method based on transformation electromagnetics. Opt. Express 2018, 26, 11708-11717. [CrossRef] [PubMed]

15. Chen, M.L.N.; Jiang, L.J.; Sha, W.E.I. Artificial perfect electric conductor-perfect magnetic conductor anisotropic metasurface for generating orbital angular momentum of microwave with nearly perfect conversion efficiency. J. Appl. Phys. 2016, 119, 064506. [CrossRef]

16. Chen, M.; Jiang, L.J.; Sha, W.E.I. Ultrathin Complementary Metasurface for Orbital Angular Momentum Generation at Microwave Frequencies. IEEE Trans. Antennas Propag. 2017, 65, 396-400. [CrossRef]

17. Zhang, L.; Mei, S.T.; Huang, K.; Qiu, C.W. Advances in Full Control of Electromagnetic Waves with Metasurfaces. Adv. Opt. Mater. 2016, 4, 818-833. [CrossRef]

18. Yu, S.X.; Li, L.; Shi, G.M.; Zhu, C.; Zhou, X.X.; Shi, Y. Design, fabrication, and measurement of reflective metasurface for orbital angular momentum vortex wave in radio frequency domain. Appl. Phys. Lett. 2016, 108, 121903. [CrossRef]

19. Yu, S.X.; Li, L.; Shi, G.M. Dual-polarization and dual-mode orbital angular momentum radio vortex beam generated by using reflective metasurface. Appl. Phys. Express 2016, 9, 082202. [CrossRef]

20. Yang, J.; Zhang, C.; Ma, H.F.; Zhao, J.; Dai, J.Y.; Yuan, W.; Yang, L.X.; Cheng, Q.; Cui, T.J. Generation of radio vortex beams with designable polarization using anisotropic frequency selective surface. Appl. Phys. Lett. 2018, 112, 203501. [CrossRef]

21. Yu, S.X.; Li, L.; Shi, G.M.; Zhu, C.; Shi, Y. Generating multiple orbital angular momentum vortex beams using a metasurface in radio frequency domain. Appl. Phys. Lett. 2016, 108, 241901. [CrossRef] 
22. Zhang, C.; Deng, L.; Zhu, J.; Hong, W.; Wang, L.; Yang, W.; Li, S. Control of the Spin Angular Momentum and Orbital Angular Momentum of a Reflected Wave by Multifunctional Graphene Metasurfaces. Materials 2018, 11, 1054. [CrossRef] [PubMed]

23. Wang, L.; Shi, H.; Zhu, S.; Li, J.; Zhang, A.; Li, L. Generation of multiple modes microwave vortex beams using tunable metasurface. In Proceedings of the 2017 7th IEEE International Symposium on Microwave, Antenna, Propagation, and EMC Technologies (MAPE), Xi'an, China, 24-27 October 2017; pp. 379-381.

24. Jiang, Z.H.; Kang, L.; Hong, W.; Werner, D.H. Highly Efficient Broadband Multiplexed Millimeter-Wave Vortices from Metasurface-Enabled Transmit-Arrays of Subwavelength Thickness. Phys. Rev. Appl. 2018, 9, 064009. [CrossRef]

25. Chen, M.L.N.; Jiang, L.J.; Sha, W.E.I. Orbital Angular Momentum Generation and Detection by Geometric-Phase Based Metasurfaces. Appl. Sci. 2018, 8, 362. [CrossRef]

26. Bliokh, K.Y.; Bekshaev, A.Y.; Nori, F. Dual electromagnetism: Helicity, spin, momentum and angular momentum. New J. Phys. 2013, 15, 033026. [CrossRef]

27. Liu, Y.J.; Xia, S.; Shi, H.Y.; Zhang, A.X.; Xu, Z. Dual-band and high-efficiency polarization converter based on metasurfaces at microwave frequencies. Appl. Phys. B-Lasers Opt. 2016, 122, 178. [CrossRef]

28. Dong, G.X.; Shi, H.Y.; Xia, S.; Li, W.; Zhang, A.X.; Xu, Z.; Wei, X.Y. Ultra-broadband and high-efficiency polarization conversion metasurface with multiple plasmon resonance modes. Chin. Phys. B 2016, 25, 084202. [CrossRef]

29. Ran, Y.Z.; Liang, J.G.; Cai, T.; Li, H.P. High-performance broadband vortex beam generator using reflective Pancharatnam-Berry metasurface. Opt. Commun. 2018, 427, 101-106. [CrossRef]

(C) 2018 by the authors. Licensee MDPI, Basel, Switzerland. This article is an open access article distributed under the terms and conditions of the Creative Commons Attribution (CC BY) license (http:/ / creativecommons.org/licenses/by/4.0/). 